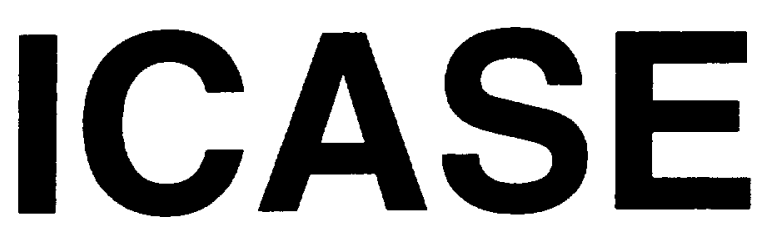

\title{
THE FAVRE - REYNOLDS AVERAGE DISTINCTION AND A CONSISTENT GRADIENT TRANSPORT EXPRESSION FOR THE DISSIPATION
}

\section{J. R. Ristorcelli \\ J. H. Morrison}

NASA Contract No. NAS1-19480

March 1996

Institute for Computer Applications in Science and Engineering NASA Langley Research Center

Hampton, VA 23681-0001

Operated by Universities Space Research Association

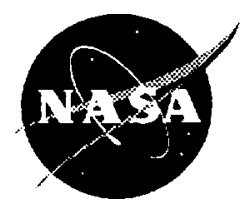

National Aeronautics and Space Administration

Langley Research Center

Hampton, Virginia 23681-0001 



\title{
The Favre - Reynolds average distinction and a consistent gradient transport expression for the dissipation
}

\author{
J.R. Ristorcelli \\ Institute for Computer Applications in Science and Engineering \\ NASA Langley Research Center, Hampton, VA
}

J.H. Morrison

Analytical Services \& Materials, Hampton, VA

\begin{abstract}
${ }^{1}$
Two equation and higher order closures for compressible turbulence fail to capture the compressible wall layers' log scaling. Accounting for the distinction between Favre and Reynolds averaged variables in the compressible moment equations indicate that turbulent transport expressions obtained using the "variable density approximation" are in error. The error is related to the enstrophy, a Reynolds averaged variable appearing in the equation for the Favre averaged $k$; recognizing this fact an expression for the transport of dissipation consistent with simple mixing length arguments is obtained. Within the (limited) context of a gradient transport hypothesis a rational form for the turbulent transport of the dissipation is found. Modestly better agreement with the well established compressible Van Driest $\log$ scaling is found in a $k-\varepsilon$ calculation.
\end{abstract}

\footnotetext{
${ }^{1}$ This research was supported by the National Aeronautics and Space Administration under NASA Contract No. NAS1-19480 while the author was in residence at the Institute for Computer Applications in Science and Engineering (ICASE), NASA Langley Research Center, Hampton, VA 23681-0001.
} 


\section{Introduction}

Huang et al. [1], Wilcox [2] have indicated that current $k-\varepsilon$ turbulence closures do not adequateley capture the compressible log laver behavior. Part of the problem with these sorts of turbulence closures appears to result from not adequately recognizing the distinction between Favre and Reynolds averaged quantities - in the Favre averaged equations both Favre and Reynolds averaged variables naturally appear. When mean density gradients are important, not accounting for the distinction contributes to poor results.

In this article a derivation for the modelled dissipation equation for a compressible turbulent flow is described. While not accounting for many of the complex effects of compressibility, discussed in a general sense in Lele [4] or in the context of the fluctuating dilatation eg. Ristorcelli [5], the present development. which involves no modeling assumptions, shows better agreement with the compressible Van Dreist log law scaling. The usual gradient transfer expression for the turbulent transport of the dissipation is found to be written as $\nu_{t} \nabla\left(\bar{\rho} \varepsilon_{s}\right)$ and not as $\nu_{t} \bar{\rho} \nabla \varepsilon_{s}$ as obtained using the so-called "variable density extension". Huang [3] has communicated that such a form for the turbulent transport will produce better agreement with the Van Driest scaling. Wilcox [2] also comments on the fact that, if $\bar{\rho} \varepsilon$ were the dependent variable, then $k-\varepsilon$ models would have closer agreement with the Van Driest scaling. While there has been speculation, Huang [1] about the properly conserved flow variable, there does not appear to be a clear indication as to what the proper formulation for the dissipation equation should be. This note indicates the appropriate form using a simple, but careful mathematical development.

\section{Derivation}

The nomenclature is now described. Upper case letters will be used to denote mean quantities and lower case letters fluctuating quantites. Exceptions to this rule are the mean density, $\bar{\rho}$, ( $\rho$ has no convenient upper case form) or the mean viscosity $\bar{\mu}$. Quantities with an asterisk denote the total field, mean and fluctuating: $\rho^{*}=\bar{\rho}+\rho^{\prime}$ or $u_{i}^{*}=U_{i}+u_{i}$. Favre velocities will be denoted using the set $\left[V_{i}, v_{i}\right]$ while Reynolds variables are denoted using the set $\left[U_{i}, u_{i}\right]$. The dependent variables are decomposed according to $u_{i}^{*}=U_{i}+u_{i}=V_{i}+v_{i}$ where $<u_{i}>=0,\left\{v_{i}\right\}=0$, and $\rho^{*}=\bar{\rho}+\rho^{\prime}$ where $\left\langle\rho^{\prime}\right\rangle=0$. The averaging operation is indicated using the angle brackets for time means, $\left\langle v_{i} v_{j}\right\rangle$, and the curly brackets for the density-weighted or Favre mean $\left\{v_{i} v_{j}\right\}$; the two averages are related by $\bar{\rho}\left\{v_{i} v_{j}\right\}=\left\langle\rho^{*} v_{i} v_{j}\right\rangle$. Without loss of clarity the prime on the fluctuating density is dropped. The portion of the second-order moment equations,

$$
\bar{\rho} \frac{D}{D t}\left\{v_{i} v_{j}\right\}=P_{i j}+\Pi_{i j}+T_{i j k, k}-<u_{j, p} \sigma_{i p}^{u}>-<u_{i, p} \sigma_{j p}^{u}>
$$

of interest are associated with the viscous dissipation type terms, where $\sigma_{i j}^{u}=\bar{\mu}\left[u_{i, j}+u_{j, i}-2 / 3 u_{q \cdot q} \delta_{i j}\right]$. 
The quantities $P_{i j}, \Pi_{i j}, T_{i j k, k}$ are, respectively, the production pressure strain and turbulent transport of the Favre averaged Reynolds stress, $\left\{v_{j} v_{j}\right\}$. Note that in the Reynolds stress equations the terms arising from the surface forces terms appear naturally in Reynolds, $u_{i}$, variables while the problem is posed in Favre, $v_{i}$, variables. Repeated application of the product rule for differentiation and the definition of vorticity $\omega_{i}=\epsilon_{i j k} u_{j, k}$ produces in the equation for the kinetic energy of the turbulence the usual dissipation quantities. Keeping only terms of interest produces

$$
\bar{\rho} \frac{D}{D t} k=-\bar{\rho}\left\{v_{i} v_{j}\right\} V_{i, j}+T_{k}-\bar{\mu}<\omega_{k} \omega_{k}>-\frac{4}{3} \bar{\mu}<d d>
$$

where $k=\left\{v_{j} v_{j}\right\}$. The solenoidal dissipation has been rewritten in terms of the enstrophy assuming small scale isotropy. The dilatational dissipation is denoted $\left.\varepsilon_{c}=\frac{4}{3}<d d\right\rangle$. Our interest is with the portion of the dissipation associated with the vortical modes of the flow. It is conventional to define the solenoidal dissipation in terms of the Reynolds averaged enstrophy: $\bar{\rho} \varepsilon_{s}=\bar{\mu}\left\langle\omega_{k} \omega_{k}\right\rangle$. In the "variable density approximation" and high Reynolds log layer limit the dissipation is assumed to be described by the high Reynolds number form of the Favre averaged equation

$$
\bar{\rho} \frac{D}{D t} \varepsilon_{s}-\left[\bar{\rho} \nu_{t} \sigma_{\varepsilon}^{-1} \varepsilon_{s, q}\right]_{q}=\bar{\rho}\left[c_{\varepsilon 1} P_{k}-c_{\varepsilon 2} \varepsilon_{s}\right] \varepsilon_{s} / k .
$$

where $P_{k}=-\left\{v_{i} v_{j}\right\} S_{i j} . S_{i j}=\frac{1}{2}\left[V_{i, j}+V_{j, i}-\frac{2}{3} D \delta_{i j}\right]$ in which $D=U_{p, p}$ is the mean dilatation which is small in the log layer portion of this flow. Note the location of the mean density in the turbulent transport term. The enstrophy's replacement by what is treated as a Favre averaged variable, $\varepsilon_{s}$, must be done carefully. The conservation equation for the enstrophy, the primitive Reynolds averaged variable appearing in the Reynolds stress equations, is, to lowest order,

$$
<\omega^{2}>_{, t}+U_{q}<\omega^{2}>_{, q}+<u_{q} \omega^{2}>_{, q}=-\frac{4}{3}<\omega^{2}>D+2<\omega_{i} s_{i j} \omega_{i}>-\bar{\mu}<\omega_{i}, \omega_{i} \omega_{k}>
$$

The last two terms in the above equations are modeled by the right hands side of equation 3 . The gradient transport hypothesis for the enstrophy transport produces $\left\langle u_{q} \omega^{2}>=-\nu_{t}\left\langle\omega^{2}\right\rangle_{q q}\right.$, where $\nu_{t}=c_{\mu} k^{2} / \varepsilon$. Replacing the enstrophy with the dissipation using $\bar{\rho} \varepsilon_{s}=\bar{\mu}\left\langle\omega_{k} \omega_{k}\right\rangle$ produces

$$
\bar{\rho} \frac{D}{D t} \varepsilon-\left[\nu_{t} \sigma_{\varepsilon}^{-1}(\bar{\rho} \varepsilon)_{q}\right]_{, q}=-\frac{1}{3} \bar{\rho} \varepsilon D+\bar{\rho}\left[c_{\varepsilon 1} P_{k}-c_{\varepsilon 2} \varepsilon_{s}\right] \varepsilon_{s} / k .
$$

The substantial derivative is along the Favre mean streamline. Note the location of the mean density in the turbulent transport term. In general the appearance of the mean density inside the first derivative will only be of importance when the mean density gradients are large, $\frac{\nabla \bar{\rho}}{\bar{\rho}} \sim \frac{\nabla \varepsilon}{\varepsilon}$. For weak density gradients the variable density assumption and the present derivation will give the same computational result. Calculations, to be shown shortly, were performed with a standard $k-\varepsilon$ model with the usual gradient transport models for transport. The following values for the empirical constants were used: $c_{\mu}=0.09, c_{\varepsilon 1}=1.44, c_{\varepsilon 2}=1.92, \sigma_{k}=1.0, \sigma_{\varepsilon}=1.17$. 
The better performance of the $k-\omega$ type of turbulence models comes from, in part, the fact that $\omega=\varepsilon / k$ is not a specific (per unit mass) variable and the Favre form of the convection operator is the correct expression for its turbulent transport.

\section{Conclusion}

One can follow Huang et al.'s [1] mean density gradient contribution analysis to asses the impact of these new forms of the transport terms in the $k$ and $\varepsilon$ equations. The coefficients multiplying the density gradient terms in their equation (20) are notably smaller using the present formulation. Wilcox [2] has conducted a similar analysis in which he arrives at a similar conclusion. The implication of both these analyses is that the deviation from the law of the wall will be smaller with these changes to the transport terms. This is substantiated in Figure 1 in which a Mach 8 boundary layer calculation using a $k-\varepsilon$ turbulence model is shown. The top line corresponds to "variable density approximation"; the lower line(s) which are virtually indistinguishable correspond to the empirical compressible log law and an incompressible calculation. The middle lines are with the new form of the transport in the dissipation equation given above: it shows a modest improvement. The upper middle line is without the bulk dilatation term. The point of this article is to present a rational form for the dissipation equation without adding ad hoc corrections. The compressible moment equations are, of course, complicated by several additional issues: the effects of the fluctuating dilatation and correlations involving the fluid property fluctuations, for example. These are the subject of current research and are expected to make additional contributions towards a better agreement with the well established Van Driest scaling.

To summarize: by careful accounting for the distinction between Favre and Reynolds averaged variables a different expression for the turbulent transport of the dissipation has been derived. The expression is consistent with mixing length arguments adequate for wall layers. Calculations for the Mach 8 bounday layer show that following a rational procedure produces a closer agreement with the log law of the wall using the conventional models for the other terms in the modeled dissipation equation.

\section{References}

[1] Huang, P.G., P. Bradshaw, T.J. Coakley (1994). Turbulence models for compressible boundary lavers. AIAA J. 32:735.

[2] Wilcox, D.C. (1993). Turbulence modeling for CFD. DCW Industries, La Canada CA.

[3] Huang, P.G. (199.5). Personal communication. 
[4] Lele, S.K. (1994). Compressibility effects on turbulence. Ann. Rev. Fluid Mech. 26:211.

[5] Ristorcelli, J.R. (1995). A pseudo-sound constitutive relationship and closure for the dilatational covariances in compressible turbulence: An analytical theory. ICASE report 95-22, submitted J. Fluid Mech. 


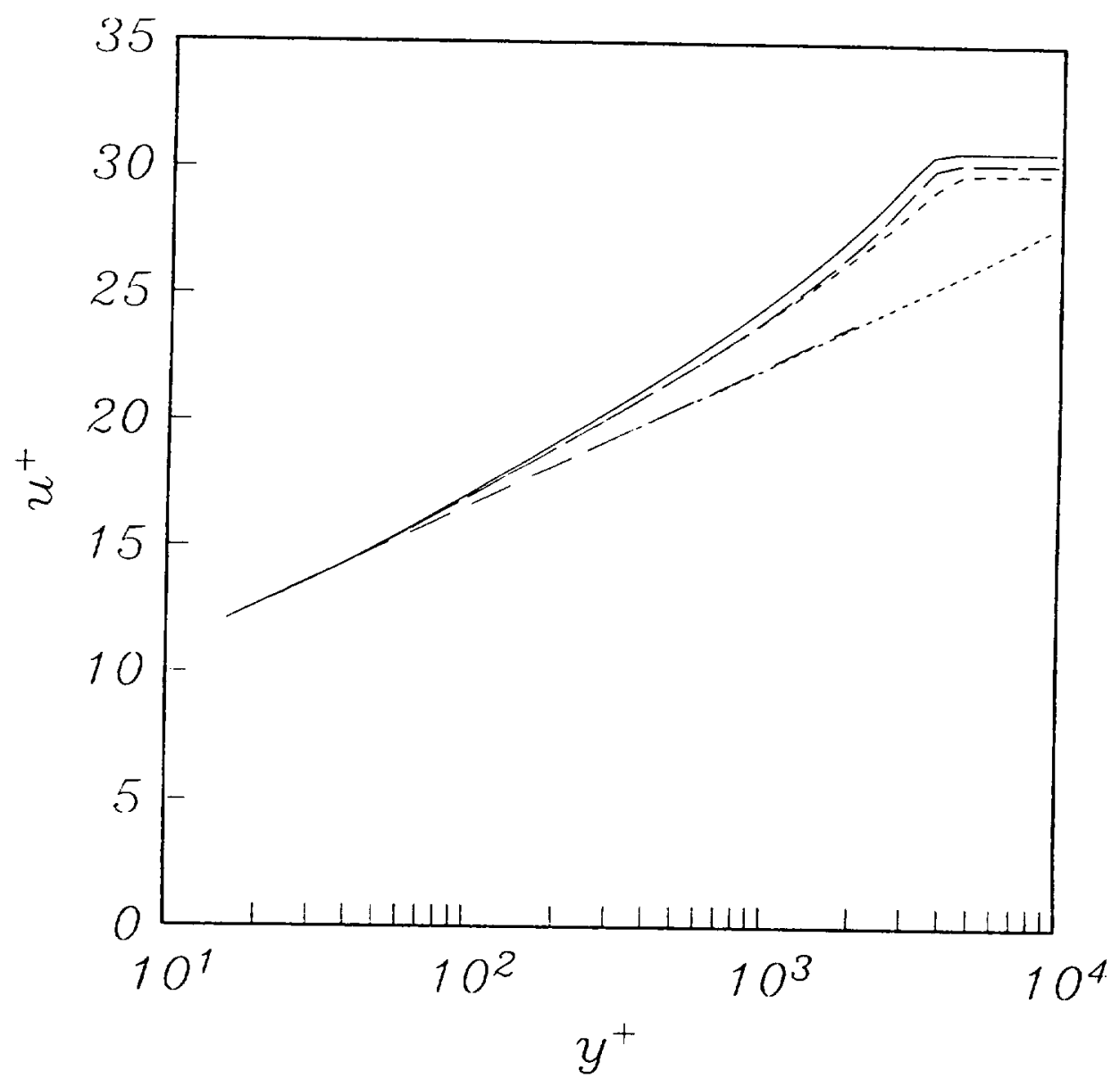

Figure 1. The mean profile for the Mach 8 boundary layer: upper line - variable density aproximation; lower lines - the log law and the incompressible calculation; middle lines - the present theory with and without (upper) bulk dilatation. 
Public reporting burden for this collection of information is estimated to average 1 hour per response, ineluding the time for reviewing instructions, searching existing data soupces. gathering and maintaining the data needed, and completing and reviewing the collection of information. Send comments regarding this burden estimate or any other aspect of this

collection of information. including susgestions for redueing this burden, to Washington Headquarters Services. Directorate for Information Operations and Reports, 1215 Jefler
Davis Highway. Suite 1204. Arlington. VA 22202-4302, and to the Office of Management and Budget. Paperwork Reduction Project (0704.0188). Washington, DC 20503.

\begin{tabular}{|c|c|c|}
\hline 1. AGENCY USE ONLY(Leave blank) & $\begin{array}{l}\text { 2. REPORT DATE } \\
\text { March } 1996\end{array}$ & $\begin{array}{l}\text { 3. REPORT TYPE AND DATES COVERED } \\
\text { Contractor Report }\end{array}$ \\
\hline
\end{tabular}

4. TITLE AND SUBTITLE

THE FAVRE-REYNOLDS AVERAGE DISTINCTION AND A CONSISTENT GRADIENT TRANSPORT EXPRESSION FOR

5. FUNDING NUMBERS

THE DISSIPATION

C NAS1-19480

AUTHOR(S)

J. R. Ristorcelli

J. H. Morrison

7. PERFORMING ORGANIZATION NAME(S) AND ADDRESS(ES)

Institute for Computer Applications in Science and Engineering

Mail Stop 132C, NASA Langley Research Center

Hampton, VA 23681-0001

WU 505-90-52-01

9. SPONSORING/MONITORING AGENCY NAME(S) AND ADDRESS(ES)

National Aeronautics and Space Administration

Langley Research Center

Hampton, VA 23681-0001

8. PERFORMING ORGANIZATION REPORT NUMBER

ICASE Report No. 96-19

10. SPONSORING/MONITORING AGENCY REPORT NUMBER

NASA CR-198305

ICASE Report No. 96-19

\section{SUPPLEMENTARY NOTES}

Langley Technical Monitor: Dennis M. Bushnell

Final Report

Submitted to Physics of Fluids.

12a. DISTRIBUTION/AVAILABILITY STATEMENT

12b. DISTRIBUTION CODE

Unclassified-Unlimited

Subject Category 34

13. ABSTRACT (Maximum 200 words)

Two equation and higher order closures for compressible turbulence fail to capture the compressible wall layers' log scaling. Accounting for the distinction between Favre and Reynolds averaged variables in the compressible moment equations indicate that turbulent transport expressions obtained using the "variable density approximation" are in error. The error is related to the enstrophy, a Reynolds averaged variable appearing in the equation for the Favre averaged $k$; recognizing this fact an expression for the transport of dissipation consistent with simple mixing length arguments is obtained. Within the (limited) context of a gradient transport hypothesis a rational form for the turbulent transport of the dissipation is found. Modestly better agreement with the well established compressible Van Driest log scaling is found in $k-\varepsilon$ calculation.

14. SUBJECT TERMS

Compressible Turbulence; Favre Average; Van Driest Scaling

15. NUMBER OF PAGES 7

16. PRICE CODE

$\mathrm{AO} 2$

17. SECURITY CLASSIFICATION OF REPORT

Unclassified OF THIS PAGE

Unclassified

19. SECURITY CLASSIFICATION OF ABSTRACT

20. LIMITATION OF ABSTRACT

Standard Form 298(Rev. 2-89) Prescribed by ANSI Std. 239-18 298.102 

